\title{
Research Paper \\ Depression and Perceived Social Support in the Elderly
}

\author{
Mehregan Emaminaeini ${ }^{1},{ }^{*}$ Maryam Bakhtiyari ${ }^{2}$, Hossein Hatami ${ }^{3}$, Soheila Khodakarim ${ }^{4}$, Robab Sahaf $^{5}$
}

1. Deputy of Health, Shahid Beheshti University of Medical Sciences, Tehran, Iran.

2. Department of Clinical Psychology, School of Medicine, Shahid Beheshti University of Medical Sciences, Tehran, Iran.

3. Department of Public Health, School of Health, Shahid Beheshti University of Medical Sciences, Tehran, Iran.

4. Department of Epidemiology, School of Health, Shahid Beheshti University of Medical Sciences, Tehran, Iran.

5. Department of Ageing, University of Social Welfare and Rehabilitation Sciences, Tehran, Iran.

Received: 23 Dec. 2016 Accepted: 29 Mar. 2017

Key words:

Elderly, Depression, Perceived social support

\section{A B STRACT}

Objectives The purpose of the study was to determine depression and perceived social support and some related factors among the elderly people in urban areas of Tehran in the area of Shahid Beheshti University of Medical Sciences.

Methods \& Materials A cross-sectional study was conducted with 580 elderly of Tehran in Shahid Beheshti University using a multistage systematic sampling method. Data were collected by trained questioners. Standard Beck depression, Zimet perceived social support and demographic questionnaires were used. Analysis was performed by Stata software. Mann-Whitney test, Kruskal-Wallis test, Spearman correlation and descriptive tests were used in this study.

Results The mean age of participants was $69.66 \pm 7.91$ years. A total of $67.53 \%$ of participants lived only with their spouses; $4.01 \%$ were illiterate and $89.69 \%$ owned at least one residential home. Health insurance coverage was for $92.38 \%$ of elderly. A total of 29.6 and $46.99 \%$ of the participants expressed their satisfaction with economic and living status, respectively.

The prevalences of depression in mild, moderate and severe levels were $17.41 \%, 25.58 \%$, and $8.88 \%$, respectively, and it was $51.87 \%$ in average. A total of $72.38 \%$ of participants reported their agreement with perceived social support. Kruskal-Wallis test implied that the levels of depression had a significant relationship with perceived social support ( $P>0.001$ ). By a correlation test, a negative linear correlation was observed between depression and perceived social support (-0.388). The marital status, home ownership, health insurance, and of life and economic satisfaction showed a significant relationship with both perceived social support and depression variables. Education and depression were significantly related. But, gender and depression did not show any significant relationship. There was no significant relationship between gender and education with perceived social support.

Conclusion Depression was found to be common in the elderly. There are similar economic and familial factors that are in relation to perceived social support and depression. According to the emotional and economic problems, creating the appropriate economic, cultural and social conditions can provide a healthier environment for the elderly to live in.

\section{* Corresponding Author:}

Maryam Bakhtiyari, PhD

Address: Department of Clinical Psychology, School of Medicine, Shahid Beheshti University of Medical Sciences, Tehran, Iran.

Tel: +98 (912) 2777387

E-mail: maryam_bakhtiyari@sbmu.ac.ir 


\section{Extended Abstract}

\section{Objectives}

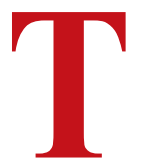

oday, approximately 580 million people of the world population are elderly people. By 2020, this figure will reach one billion [1]. Due to the reduction in mortality rate resulting from the advances in medical sciences, health and education, there has been an increase in the life expectancy rate worldwide. It is believed that there will be significant developments in a wide range of traditional structures, values, norms and the creation of social organizations. Therefore, coping with the challenges of this phenomenon and making use of appropriate measures to improve the physical, mental and social health of the elderly are of great importance and have been placed on the agenda of the international community [2]. The aim of this study, through the research conducted at Shahid Beheshti University of Medical Sciences, is to determine the status of depression, perceived social support and some related factors in the elderly persons living in Tehran.

\section{Methods \& Materials}

This cross-sectional study was conducted on 580 elderly people (60 years old and above) living in urban areas of the city of Tehran, covered by Shahid Beheshti University of Medical Sciences. Multi-stage sampling and systematic form were performed in the areas covered by all the health and treatment centers subsidiary to the health centers of the North and East regions in Tehran and Shemiranat (county located in northern part of the city of Tehran). The third residential building unit from the right side of each health and treatment center was selected as the starting point for sampling, and then all the houses of this unit were examined on all floors with regard to the presence of the elderly in those apartments. And with the same order (third residential building unit, third residential building unit), apartments were selected and questionnaires continued.

Since the ability of the research samples for filling out all of the questionnaires were in doubt, a preliminary study was conducted; its results showed that the elderly were rather weak in completing the questionnaires by themselves. Accordingly, the research samples were informed that in case they were inclined they could be referred to the health and treatment centers to complete the questionnaires. For this purpose, a number of employees were trained in each of the health and treatment centers. Given that many tools were used, the questionnaires remained with the elderly for one day to be completed. Those of the elderly who were not at home at that time or were reluctant to participate in the research were excluded from the study, and the elderly residents in the first residential unit on the right side of that unit were substituted in their place.

Suffering from neurological illnesses or known psychological disorders and a history of having been hospitalized due to psychological illnesses were the criteria for exclusion from the study. Data were collected using standard questionnaires of Beck's Depression Inventory with 13 questions and Zimet's Multidimensional Scale of Perceived Social Support in three dimensions and 12 questions and a demographic information questionnaire that had been prepared by the authors. Data were analyzed using Stata software and the descriptive and analytic statistical methods (Mann Whitney and Kruskal-Wallis and Spearman correlation test). This research is approved by the Ethics Committee of Shahid Beheshti University of Medical Sciences.

\section{Results}

Some of the elderly persons who participated in this study did not respond to certain questions; therefore, they were excluded from the calculations. By analyzing the data, it was known that the number of female participants was 348(60.52\%), number of male participants was $227(32.48 \%)$, and the average age was $69.66 \pm 7.91$ years. Among the total sample subjects, 401 persons (69.37\%) were married and $177(30.63 \%)$ were widows or widowers or had separated from their spouse.

Only $67.53 \%$ of the participants were living with their spouse. Of the total sample, $4.01 \%$ were illiterate. A total of $145(42.76 \%)$ participants had high school studies or high school diploma. $89.69 \%$ of the participants owned at least one residential quarter, and $92.38 \%$ had health insurance coverage. $46.99 \%$ of the participants expressed satisfaction with their life; however, only $29.6 \%$ were estimated to be content financially. Considering that the maximum score for each dimension of perceived social support based on Zimet's standard questionnaire was 20, the median score for the perceived social support in the family dimension was calculated to be 15.52 with the standard deviation of 4.56. In the friends dimension, the median score was 12.36 with the standard deviation of 5.22 , and in the specific people dimension, the median score was 14.69 with the standard deviation of 4.75 .

The median score for perceived social support in total (all three dimensions) was 42.71 with the standard deviation of 12.80 (out of 60 points maximum score for this questionnaire). Findings confirmed that among the elderly participating in this research, the median score of 
perceived social support in the family dimension was higher than the other two dimensions. Also, the findings showed that the prevalence of slight, mild and severe depression was $17.41 \%, 88.58 \%$ and $8.25 \%$, respectively, with a mean value of $51.87 \%$. The agreement with perceived social support was $72.38 \%$. The Kruskal-Wallis test showed that different levels of depression had a statistically significant correlation with perceived social support $(\mathrm{P}<0.001)$. Spearman's correlation test also showed a linear correlation between social support and depression stating that an increase in social support led to a decrease in the depression level (-0.388).

Marital status variables, housing ownership, health insurance, life satisfaction and economic satisfaction showed a significant correlation with both variables of depression and social support. Education had a statistically significant connection only with depression. However, there was no significant correlation between gender of the participants and depression, and also between education and gender with perceived social support.

\section{Conclusion}

In the elderly, factors related to perceived social support and depression are often common. It is likely that these two variables are somehow related to each other or may be the cause of creating each other. These common factors include being married and all the factors related to the economic status and satisfaction from life. Satisfaction from life may also be connected to economic satisfaction. Further studies need to be conducted in this regard. Social support as a component affecting psychological health and morale of the elderly can be considered as an inexpensive source and as a social capital in terms of the dynamics and improvement of the quality of life and morale of the elderly. Providing part of the physical and mental needs to the elderly can lead to an increase in the perceived social support. Unlike some studies, this research did not show a difference in the prevalence of depression and perceived social support between women and men. Therefore, the needs of elderly people should not be neglected in this regard.

\section{Acknowledgments}

This paper is extracted from the MPH thesis of the third author in the Department of Public Health, School of Health, Shahid Beheshti University of Medical Sciences, Tehran.

\section{Conflict of Interest}

All authors certify that this manuscript has neither been published in whole nor in part nor being considered for publication elsewhere. The authors have no conflicts of interest to declare. 


\title{
بررسى وضعيت افسردتى، حمايت اجتماعى ادر اكشده و برخى عوامل مرتبط با آنها در سالمندان

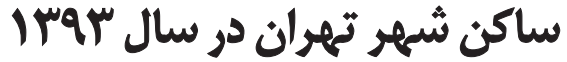

\author{
مهركان امامى نايينى'، "مريم بختيارى"، حسين حاتمى"، سهيلا خداكريم"، رباب صحافه

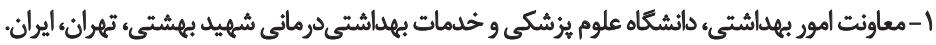

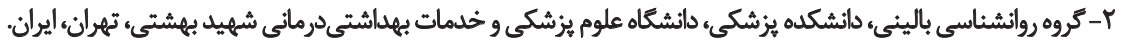

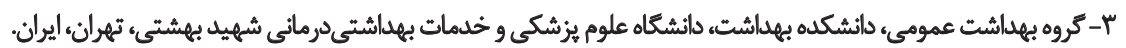

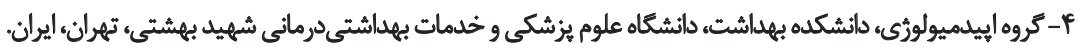

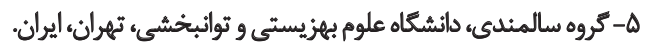

\begin{abstract}
حكبد

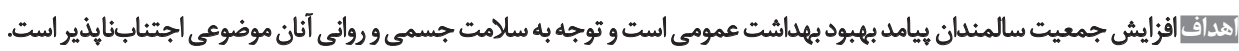

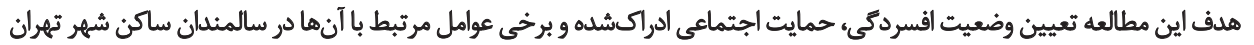

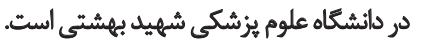

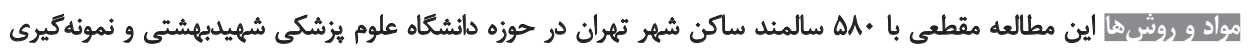

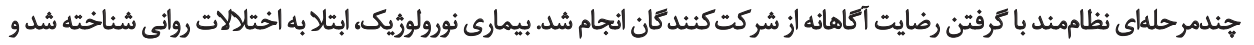

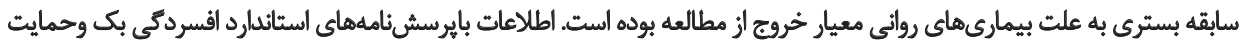

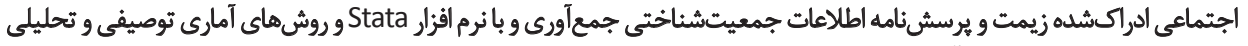

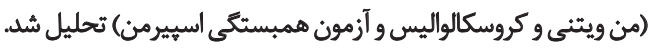

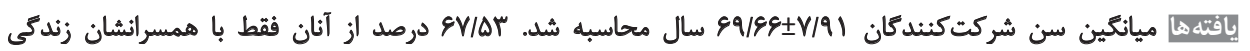

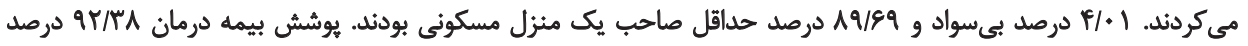

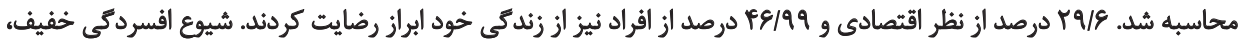

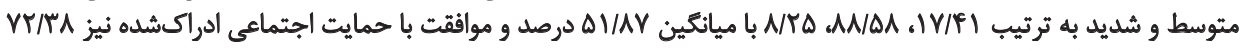

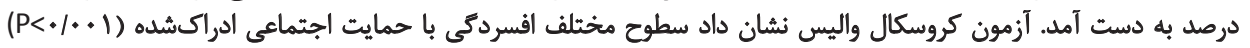

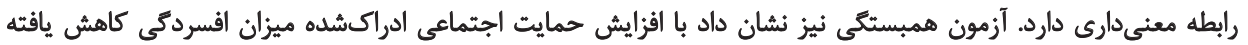

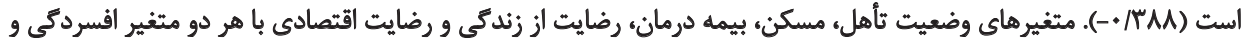

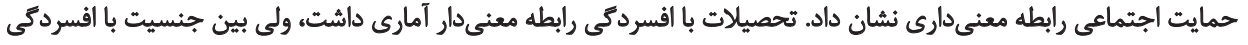

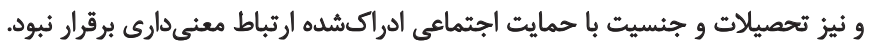

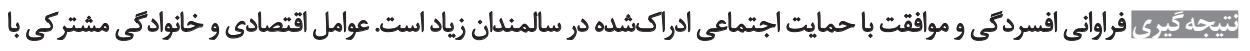

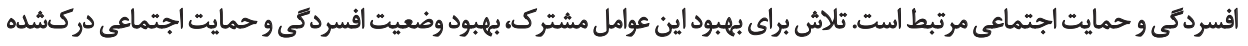

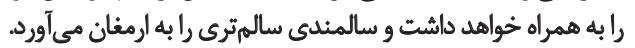

تاريخ دريافت: ب• دى هوبا

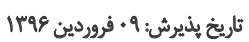

بيش از •ع سال تشكيل دادهاند [ب]. در سال •ه •r جمعيت

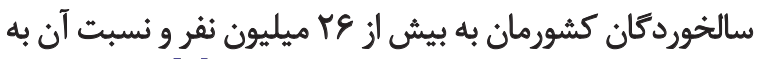

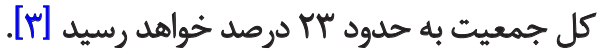
مسئله سالمندى جمعيث جهان به دلايل مهمى ازجمله

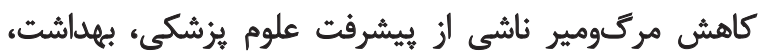

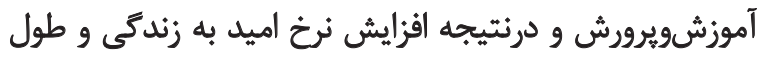

dolos

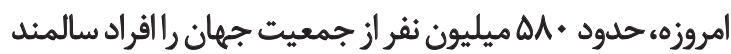

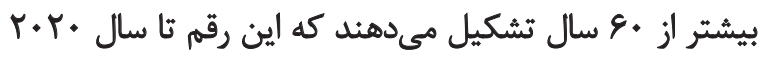

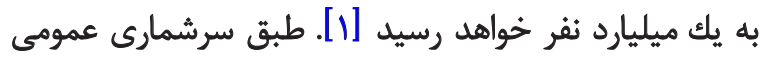

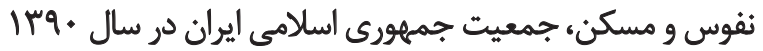

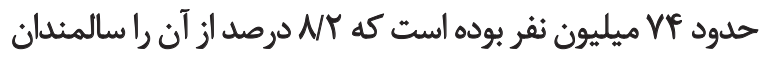

: نويسئده مسئول:

دكتر مريم بختيارى نويندان

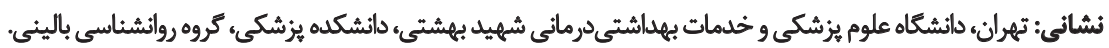

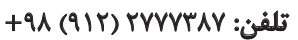
maryam_bakhtiyari@sbmu.ac.ir بهيث الكترونيكى 


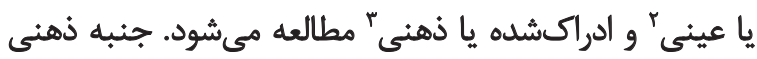

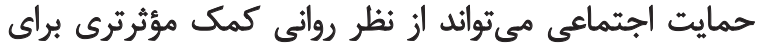

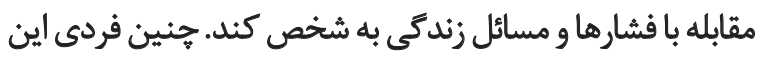

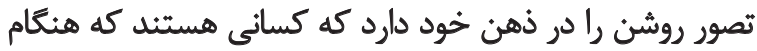

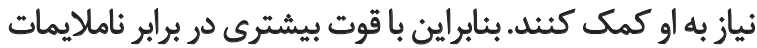

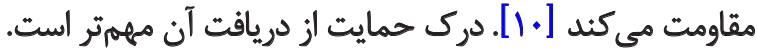

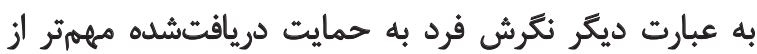

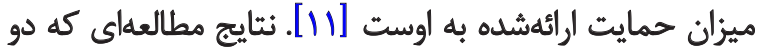

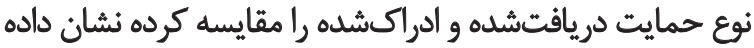

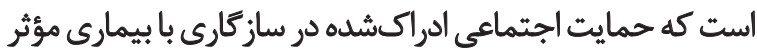

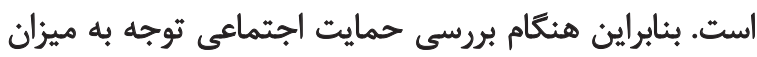
درك از آن اهميت خاصى دارد.

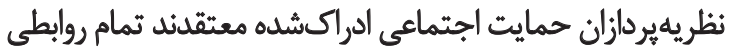

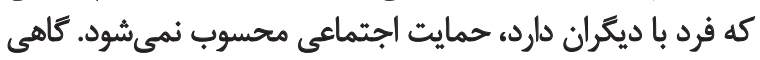

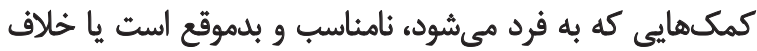

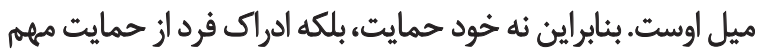

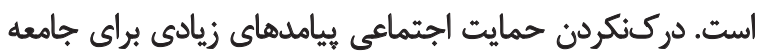

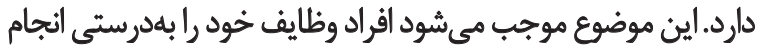

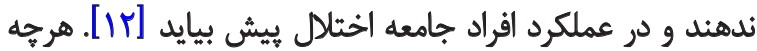

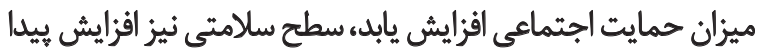

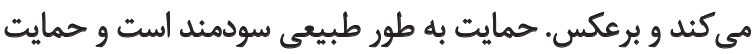

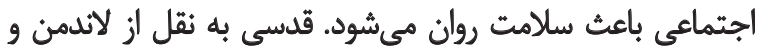

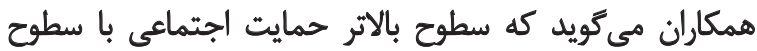

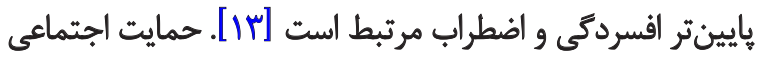

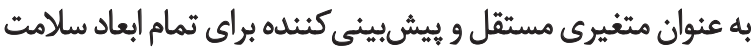

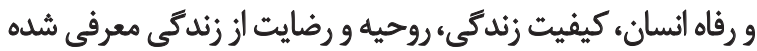

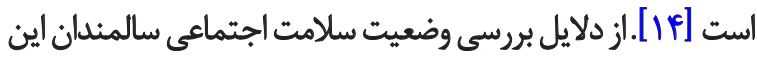

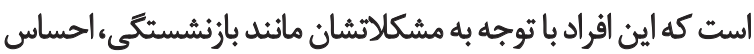

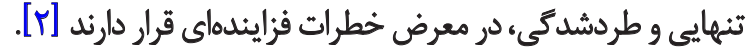

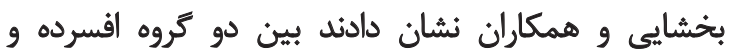

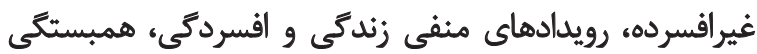

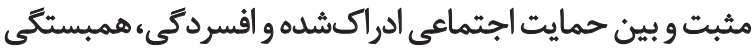

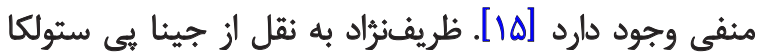

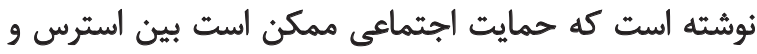

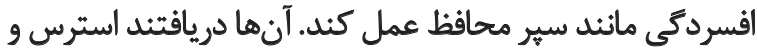

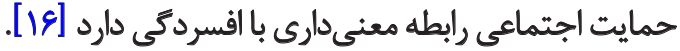
مطالعات نشان مي دهد كه حمايت اجتماعي نقش بسيار مهيهى

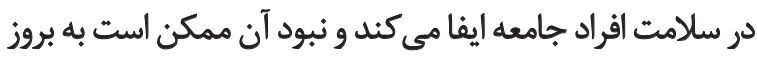

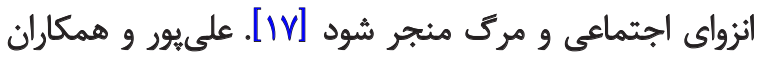

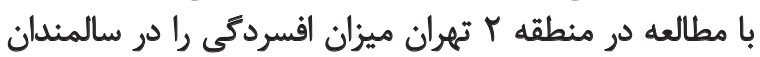

2. Revised social support

3. Perceived social support

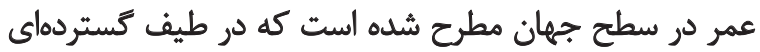

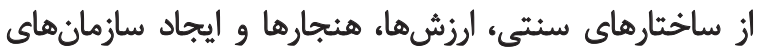

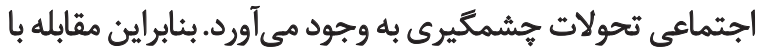

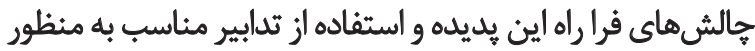

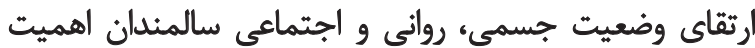

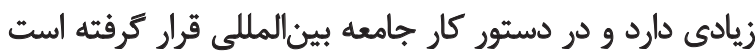

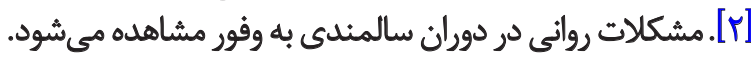

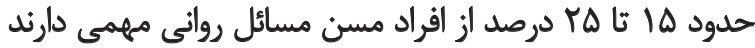

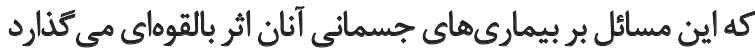

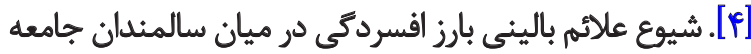

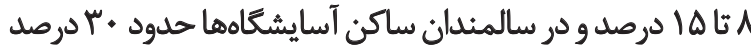

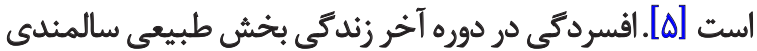

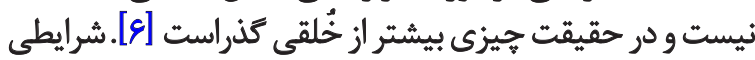

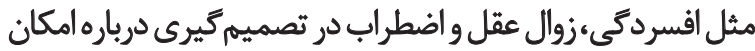

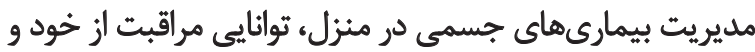

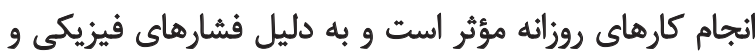

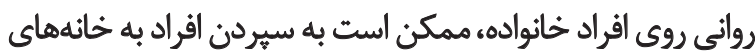

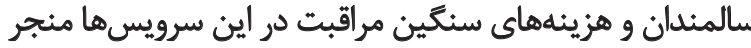
شود [ه]] بُعد رواثى يكى از ابعاد مهم سلامت سالمندان است و بـه

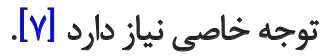

سالمند در ارتباطات اجتماعى خود ناكزير است باسو في فقدانهاي

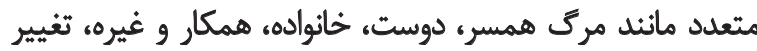

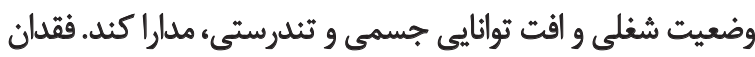

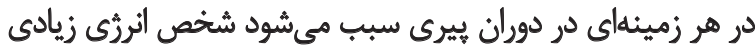

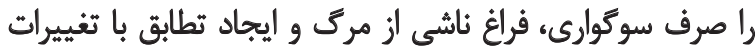

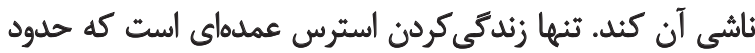

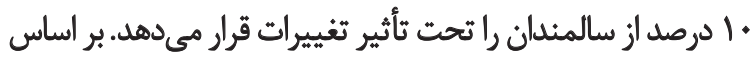

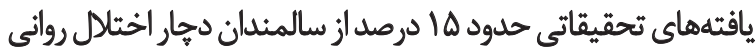

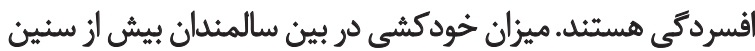

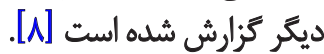

اكرجه هراقبت، دوستى و حمايت مفاهيمي به قدمت ارتباطات

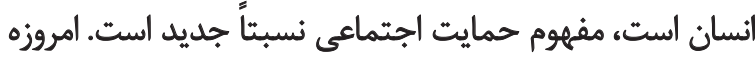

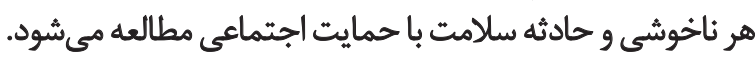

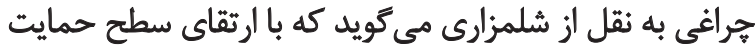

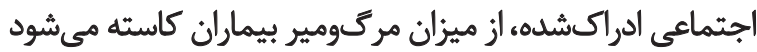

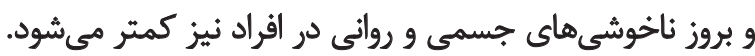

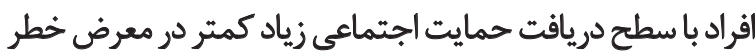

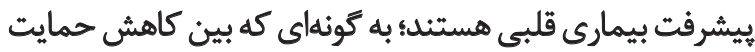

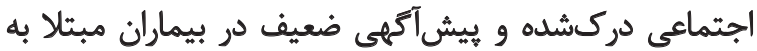

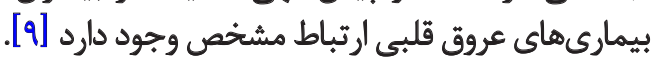
حمايت اجتماعى' به دو صورت حمايت اجتماعى دريافتشده 


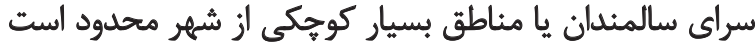

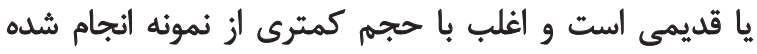

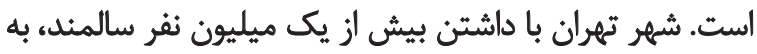

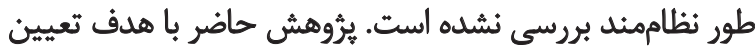

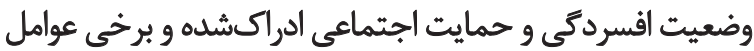
مرتبط با آن در سالمندان انجام شد.

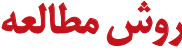

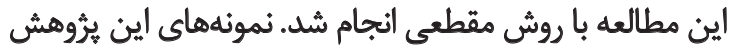

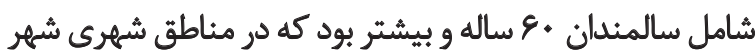

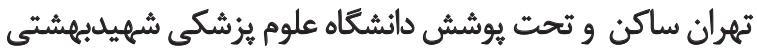

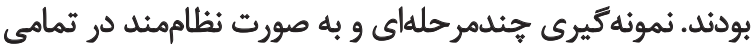

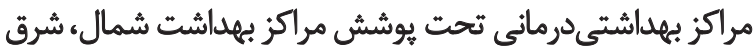

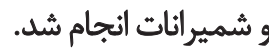

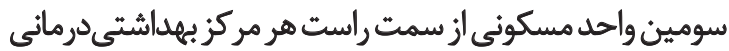

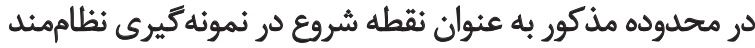

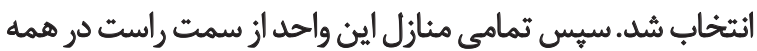

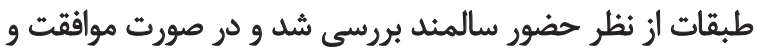

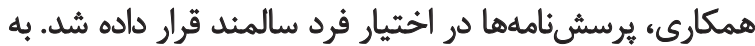

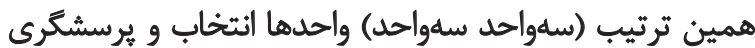

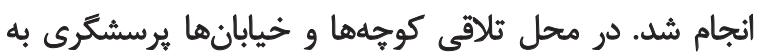

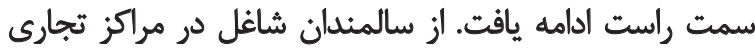

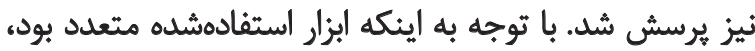

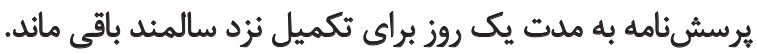

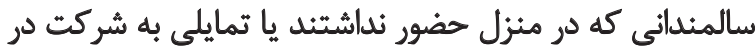

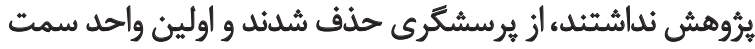

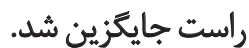

شايان ذكر است كه يرسشنامه افسردتى بك خودتكميل

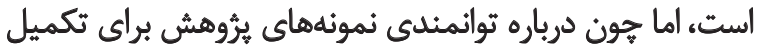

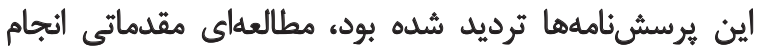

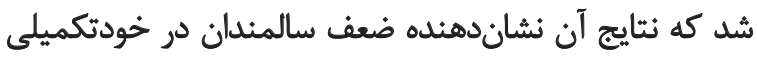

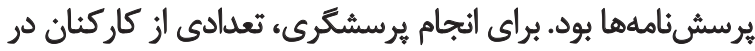

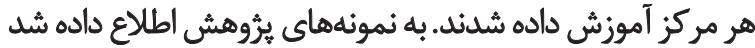

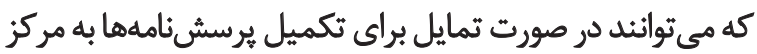

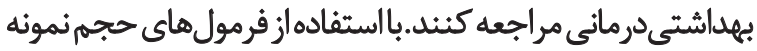

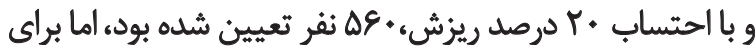

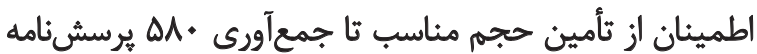

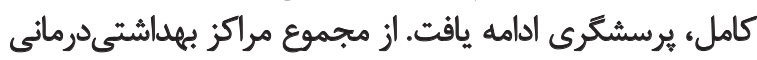

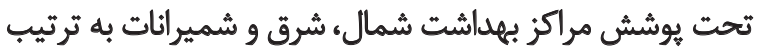

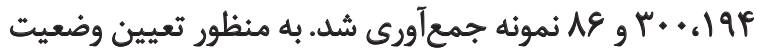

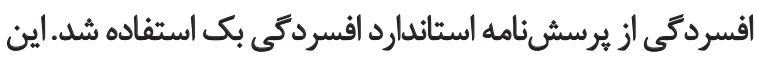

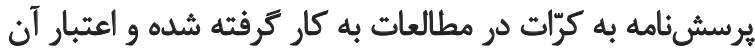

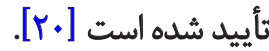

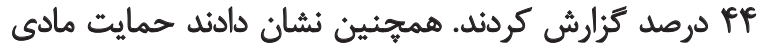

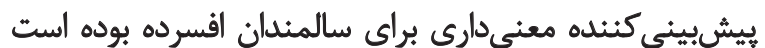

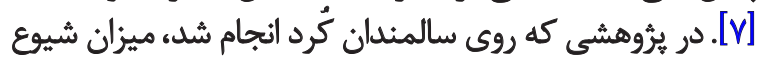

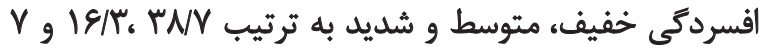

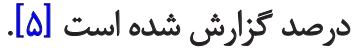

در مطالعهاي در ثايوان عوامل خطر ابتلا به افسردگى شئ شديد،

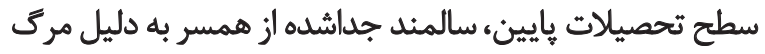

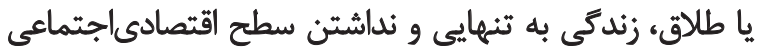

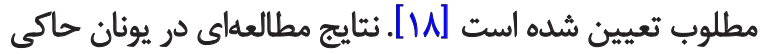

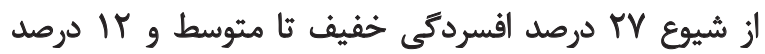

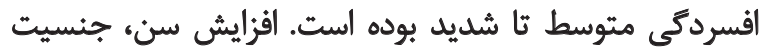

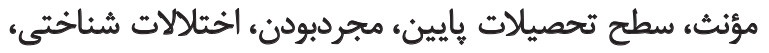

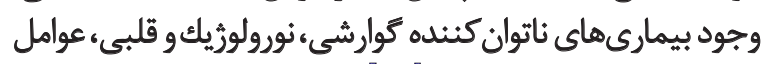
خطر ابتلا به افسردگى است [19]. نتايج بسيارى از ئوهش هاى انجامشده در ايران و جهان نشانٍ

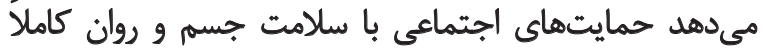

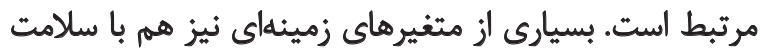

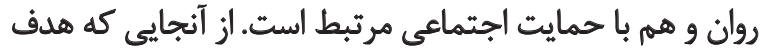

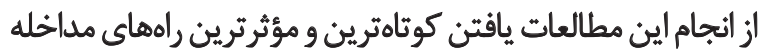

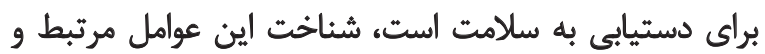

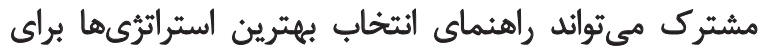
دستيابي به سلامت و كسترش آن باشدي

اكرجه تشابهات فراوانى بين يافتههاى اين مطالعات وجود

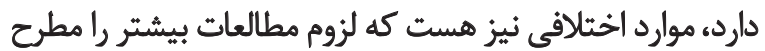

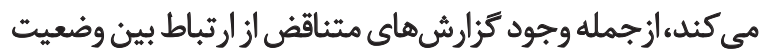

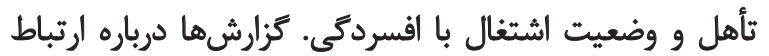

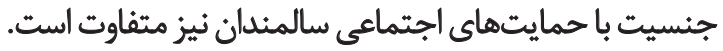

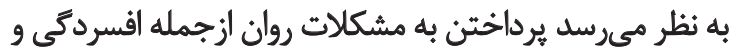

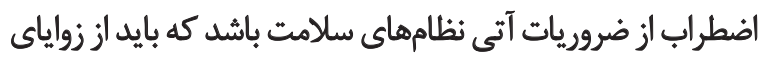

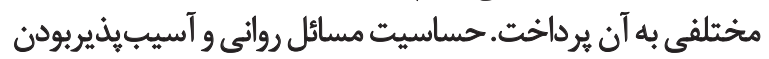

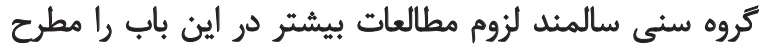

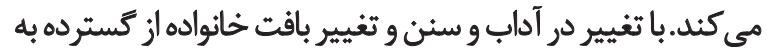

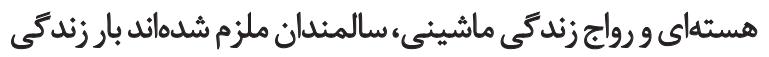

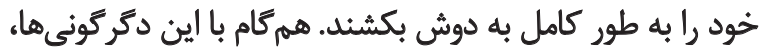

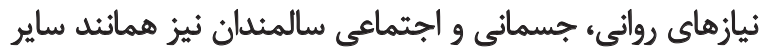

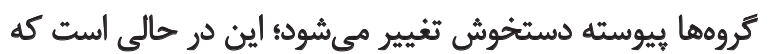

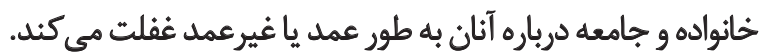

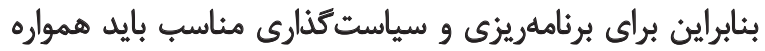

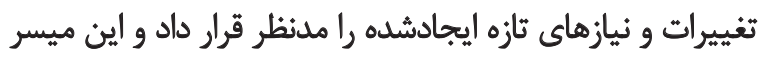

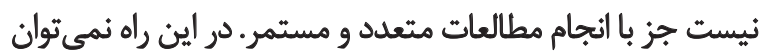
به انجام بررسى هاى يراكنده و قديمى موجود بسنده كرد.

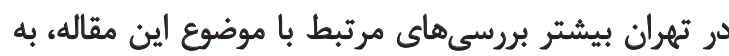


زئدكى، رضايت اقتصادى و غيره بود.از تمامى شركت كنيندكان در

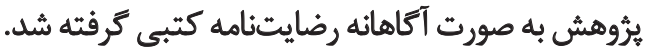

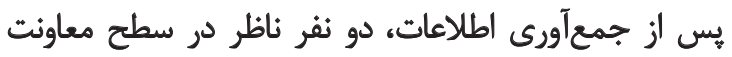

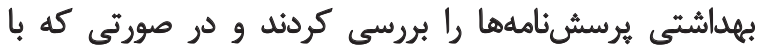

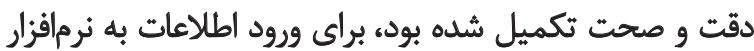

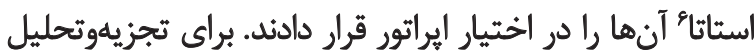

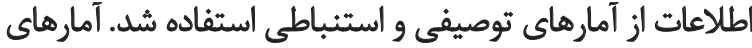

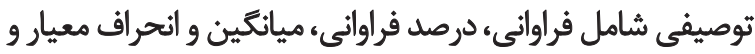

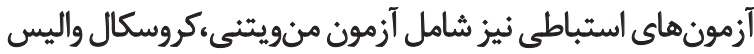

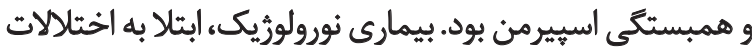

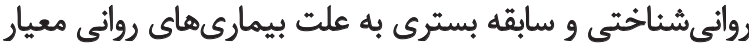
خروج از مطالعه بوده است.

ياقتهلها

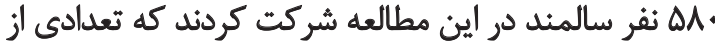

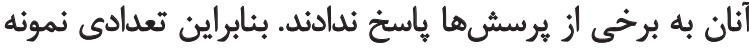

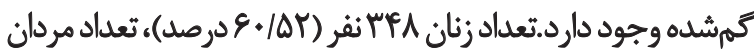

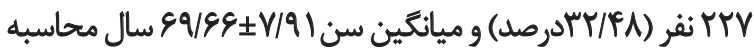

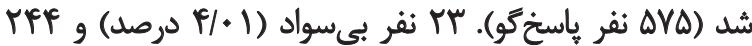

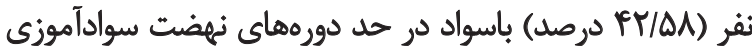

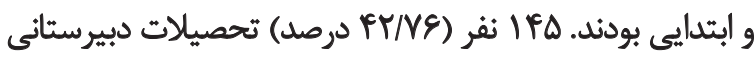

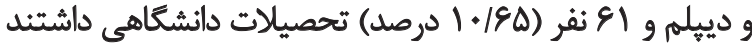

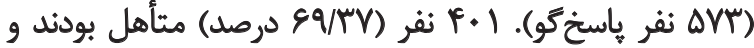

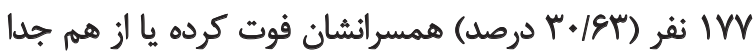

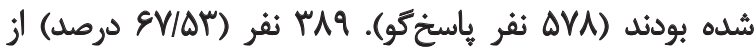

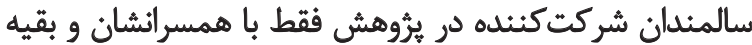

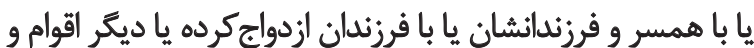

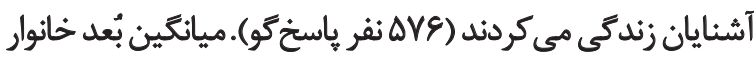

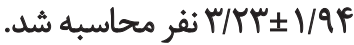

بررسى وضعيت مالكيت مسكن نشان ميدهد

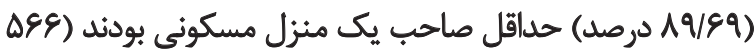

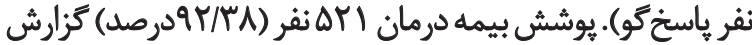

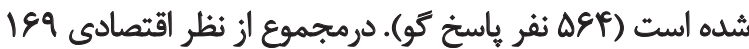

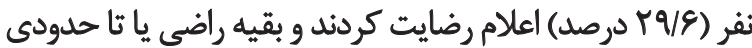

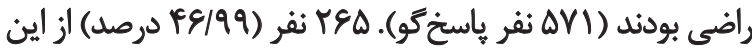

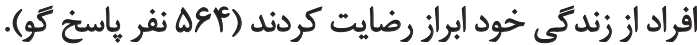
وضعيت افسردكى در سالمندان مطالعهشده به تفكيك سطوح

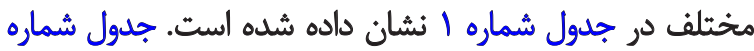

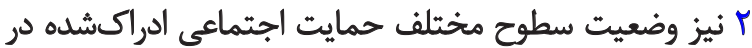

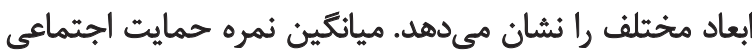

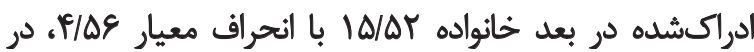

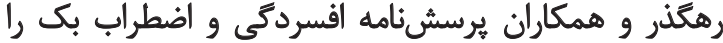

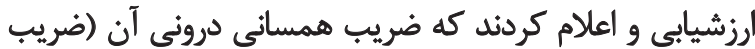

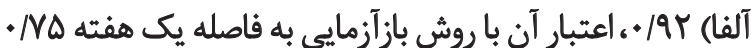

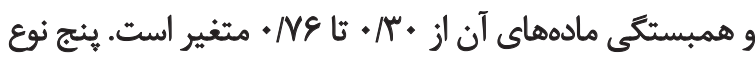

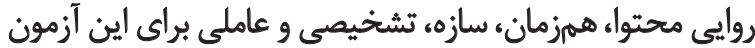

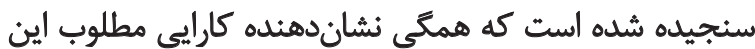

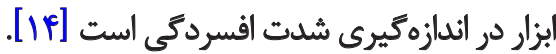

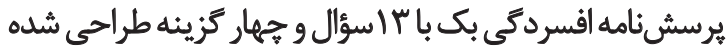

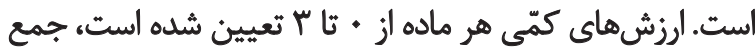

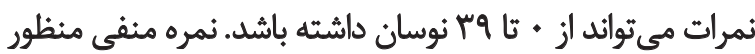

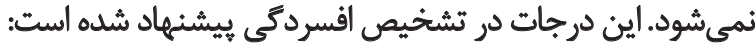

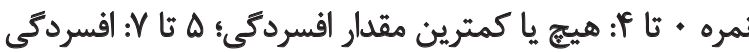

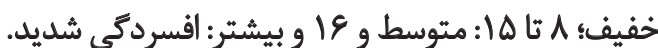

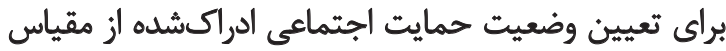

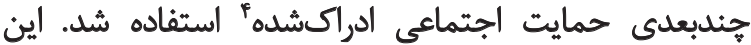

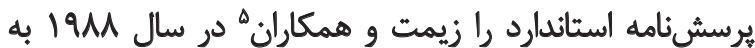

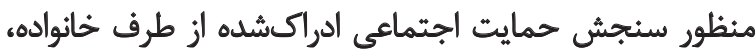

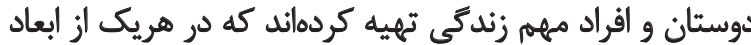

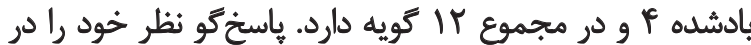

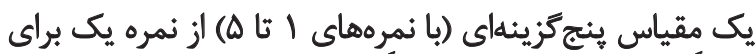

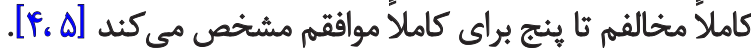

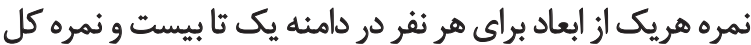

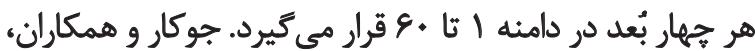

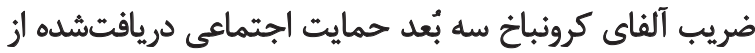

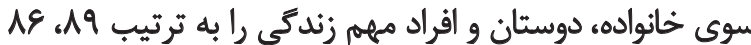

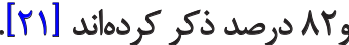

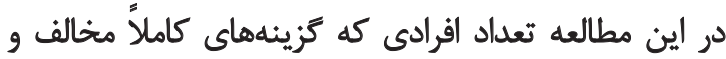

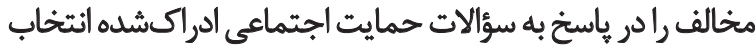

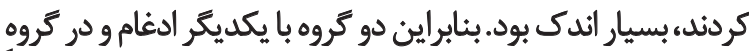

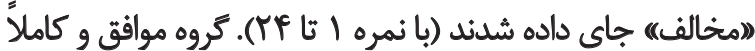

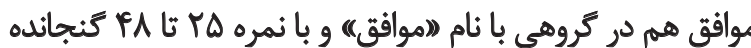

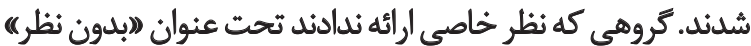

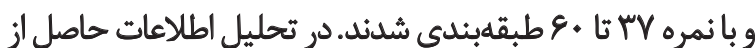

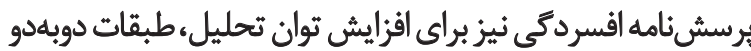

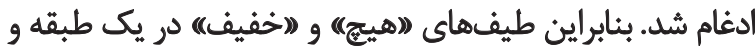
"(متوسط) و (شديد) نيز در طبقه دوم قرار داده شد.

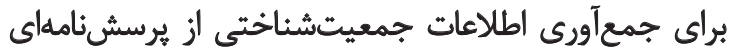

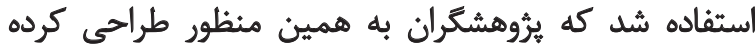

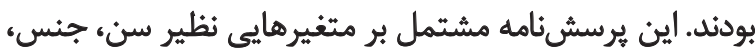

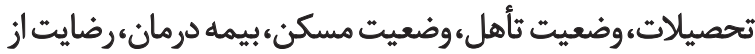

4. Multidimensional Scale of Perceived Social Support (MSPSS) 5. Zemet et al. 


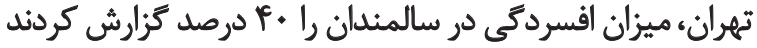

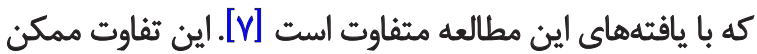
است به علت تفاوت در ابزار اندازهكيرى ،حجم نمونه يأن يا منطقه سكونت و وسعت آن باشيد.

مطالعه حاضر ارتباط معنى دارى را بين جنسيت و افسردگى مئى

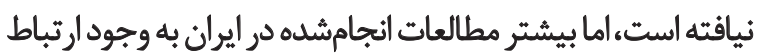

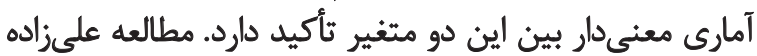

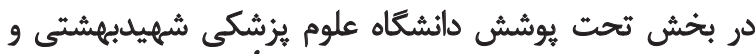

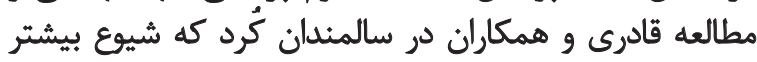

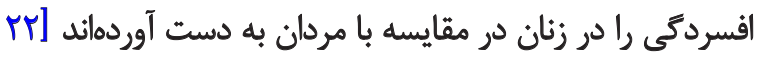

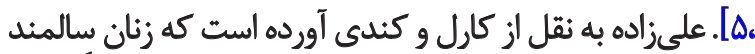

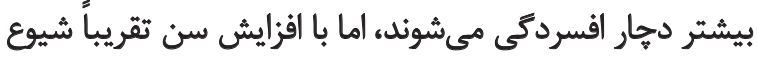

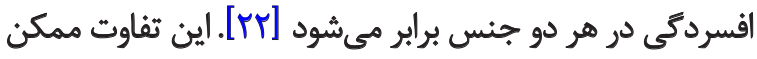

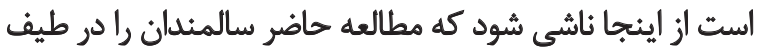

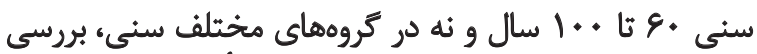

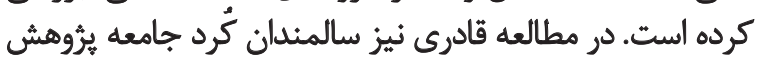

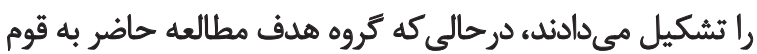

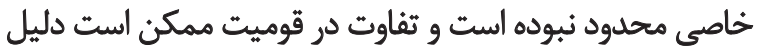

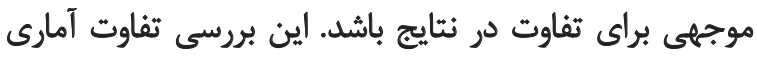

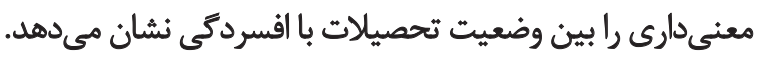

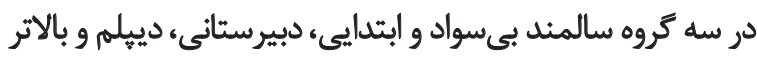

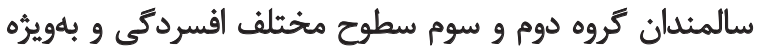

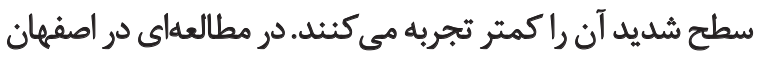

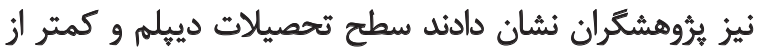

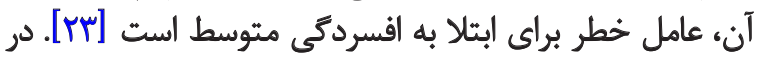

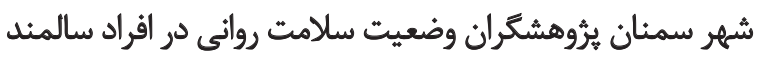

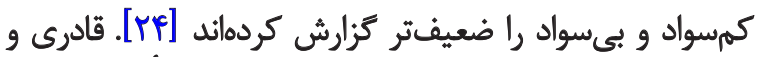

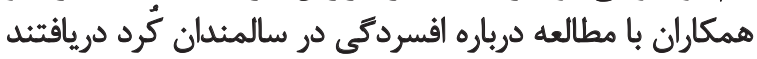

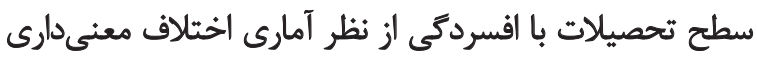

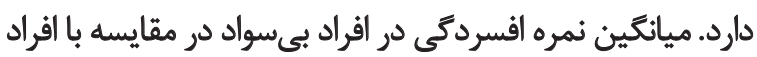

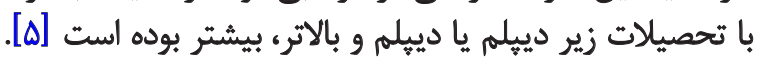

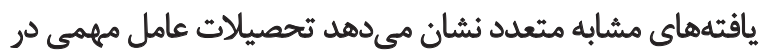

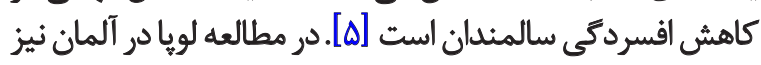

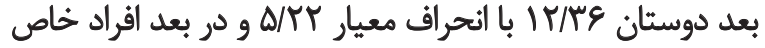

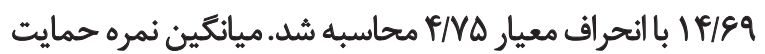

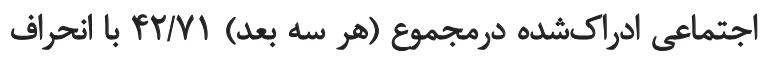

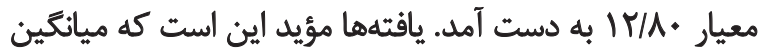

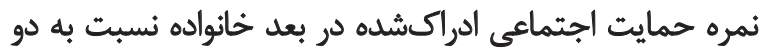

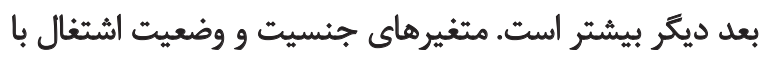
افسردگى رابطه معنى دار آمارى نشان نداد.

در بررسى وضعيت حمايت اجتماعى ادراكشده، وضعيت

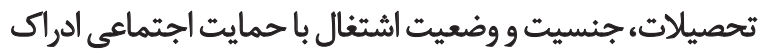

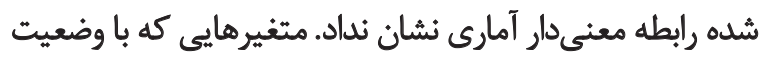

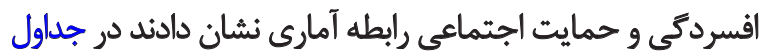

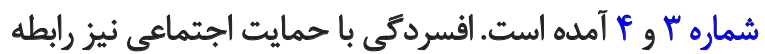

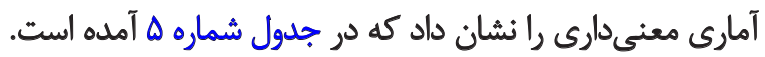

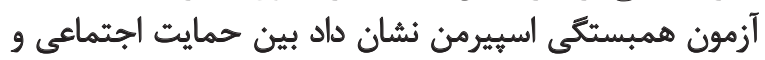

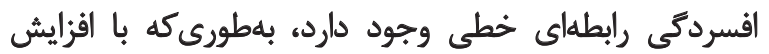

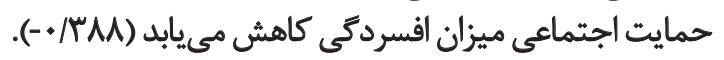

$\leftrightarrow$

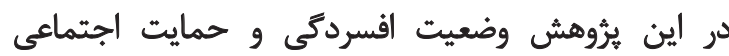

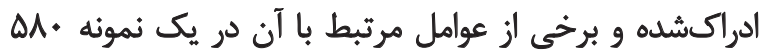

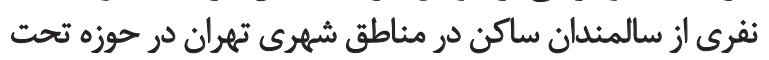

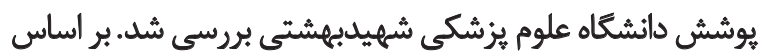
تجزيهوتحليل دادهها موارد زير قابل بحث استيل:

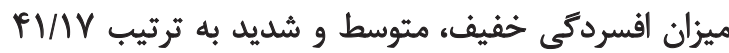

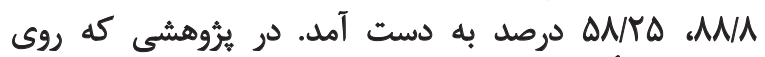

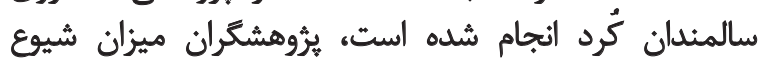

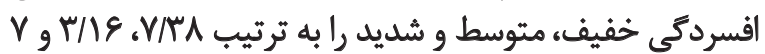

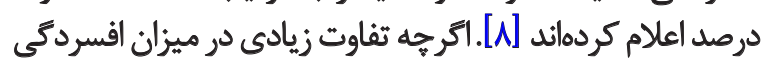

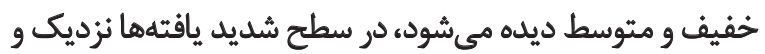

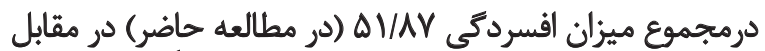

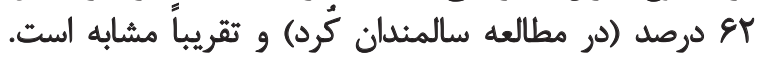

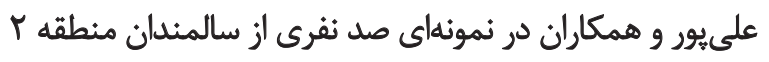

جدول ا. توزيع فراوائى مطلق و نسبى وضعيت افسردگى در سالمندان ساكن در مناطق شهرى تهران درسال بهوبا

\begin{tabular}{|c|c|}
\hline درصد (تعداد) & وضعيت افسردكى \\
\hline FNIT(TYI) & هيج يا كمترين \\
\hline$|V / F|(Q)$ & خفيف \\
\hline$\left.r \Delta / \Delta A(1 F)^{n e}\right)$ & متوسط \\
\hline$N M(\Delta \cdot)$ & شديد \\
\hline DI/AV(19Y) & هيزان افسردكى \\
\hline
\end{tabular}

$\stackrel{2}{2}$ 
جدول ז. توزيع فراوائى مطلق و نسبى سطوح حمايت اجتماعى ادراكشده در سالمندان ساكن در مناطق شهرى تهران در سال بوسا

\begin{tabular}{|c|c|c|c|c|}
\hline افرادخاص & دوستان & خانواده & حمايت اجتماعى كلى & \\
\hline وروصد (تعداد) & دوصد (تعداد) & مرصد (تعد/د) & قروصد (تعداد) & شده \\
\hline$F / N E(T V)$ & $1 . / 1 Y(\Delta \mathrm{A})$ & $F / \Delta V(Y \varepsilon)$ & $e n \cdot(\pi)$ & كاملأ مخالف \\
\hline$\Delta / A Y(M T)$ & $\mid \& / \Delta A(q \Delta)$ & $r / m(19)$ & $\left.r / \Delta \cdot(1)^{\circ}\right)$ & هخخالف \\
\hline Weq(1.8) & r)/9q(ITE) & $1 . M r(81)$ & $r V / r(I I A)$ & بلدون نظر \\
\hline$\pi T / \Delta 1(19)$. & MI/TP(IVq) & $r V / P r(T / Y)$ & $P+/ T A(T K E)$ & موافق \\
\hline MV/TI(TII) & $r+1 \cdot V(110)$ & $P T / Q Y(Y \Delta \cdot)$ & $M /+q\left(M_{*}\right)$ & خيلى مواقق \\
\hline $1 \cdots(\Delta \odot V)$ & $\cdots(\Delta V \Psi)$ & $1 \cdots(\Delta<q)$ & $1 \cdots(\Delta \& 1)$ & جمع \\
\hline
\end{tabular}

به نظر مىرسد افراد داراي سطوح بالاتر تحصيلى با برخوردارى

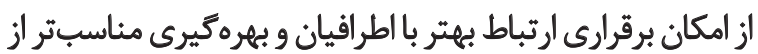

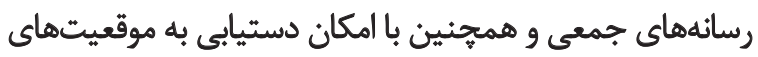

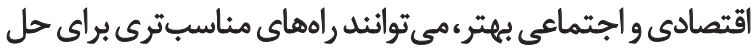

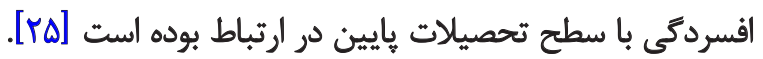

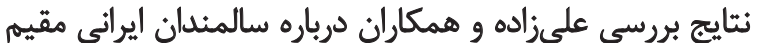

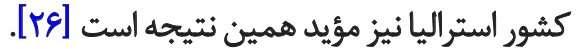

جدول ץ.ارتباط متغيرهاى جمعيتشناختى نموئهاى با وضعيت افسردگى سالمندان ساكن مناطق شهرى تهران در سال بوسبا

\begin{tabular}{|c|c|c|c|c|c|}
\hline معنى دارى & متوسط و شداديد & هرورد (تعداد) & دوصد (تعداد) & & عوامل جمعيتشناختى \\
\hline \multirow{2}{*}{. MAY } & epl.e(Irr) & $\Delta N M^{M}\left(M^{\prime} \mid P\right)$ & E./ Tq.(mY) & زن & \multirow{2}{*}{ جنسيت } \\
\hline & ro/AT(Eq) & $\left.P V E A(\mid \Delta)^{\prime}\right)$ & PQ/MI(TMY) & هرد & \\
\hline \multirow{2}{*}{$+1+\cdots+r$} & $\Delta N \cdot T(M T)$ & $V Q / \cdot V(T W V)$ & Eq/Tr(rAq) & داراى همسر & \multirow{2}{*}{ وضعيت تاهل } \\
\hline & PI / $9 Y(A)$ ) & TE/qT(qY) & $r \cdot / A V\left(I N^{2}\right)$ & جدا شده يا همسرهرده & \\
\hline \multirow{3}{*}{.1 .04} & $\Delta R / T \Delta(1 \cdot A)$ & FV/AT(MAT) & Heferf(Yel) & ابتداييى و نهضت & \multirow{3}{*}{ وضعيث تحصيلات } \\
\hline & $r \Delta / \Delta T(F q)$ & re/rA(A9) & MT/QI(ITA) & دييرستان & \\
\hline & WNr(ro) & Wr/ & $r r /+r(M M)$ & دييلم و داتشكاه & \\
\hline \multirow{2}{*}{$.1 . p q$} & $V N \cdot 1(1 F 9)$ & $A F / M r(r \cdot \Delta)$ & $A r / r q(F \Delta F)$ & دارد & \multirow{2}{*}{ مالكيت مسكن } \\
\hline & ก)/ดQ(FT) & $\mid \Delta / T N(\Delta \Delta)$ & $|V / \&|(q V)$ & ندارد & \\
\hline \multirow{2}{*}{.$/ .14$} & WTA(IE9) & $94 / \pi+(\pi+9)$ & $9 T / r g(\Delta+1)$ & دارد & \multirow{2}{*}{ بيمه درمان } \\
\hline & W/AT(YT) & $\Delta / \Delta V(T \cdot)$ & $V / A F(F)$ & ندارد & \\
\hline \multirow{3}{*}{$.1+.+r$} & 19/eq(\%1) & $r \Delta / \Lambda)(\mid r \cdot)$ & $r \cdot / r V(\mid \notin \lambda)$ & دارد & \multirow{3}{*}{ رضايت اقتصادى } \\
\hline & $\Delta r / T V(1 \cdot \Gamma)$ & $m / \cdot F(I r \cdot)$ & $P+/ M(T M T)$ & ندارد & \\
\hline & 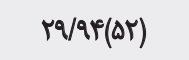 & rV/Ir(IIr) & $r Q / \Delta \Delta(I E Y)$ & تاحلودى & \\
\hline \multirow{3}{*}{$+1+\cdots+1$} & $r+18 r(r q)$ & E./TA(TIV) & PEIET(TAS) & دارد & \multirow{3}{*}{ رضايت از زندكي } \\
\hline & $r+\operatorname{lgq}(\Delta \mathrm{Al})$ & $g / T^{2}(M)$ & IYRO(A)) & ئدارد & \\
\hline & PNEN(9Y) & $M T / M T(I r \cdot)$ & TNET(TIT) & تاجلوودى & \\
\hline
\end{tabular}

先 
جدول F. ارتباط متغير هاى جمعيتشئاختى با وضعيت حمايت اجتماعى ادراكشده سالمندان ساكن در مناطق شهرى تهران درسال بهوبا

\begin{tabular}{|c|c|c|c|c|c|c|}
\hline \multirow{2}{*}{ معنىدارى } & موافق & بدون نظر & مخالف & جمع & حمايت اجتماع & \multirow[b]{2}{*}{ عوامل جمعيتشناختى } \\
\hline & درصد (تعداد) & درصد (تعداد) & درصد (تعداد) & درصد (تعداد) & & \\
\hline \multirow{2}{*}{. IAfF } & SI/Eq(MYA) & $8 Y /+r(M)$ & $\Delta F /+\Delta\left(r_{+}\right)$ & $81 / F(M+)$ & زن & \multirow{2}{*}{ جئسيت } \\
\hline & $r q / T)(\mid \Delta F)$ & rNQA(FE) & $P \Delta / Q \Delta(I V)$ & rNQE(TIV) & مرد & \\
\hline \multirow{2}{*}{ 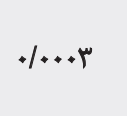 } & $V^{4} /$ / & $Q 1 / . Y(M)$ & $\Delta) / \Delta(19)$ & EA/EF(YQ.) & داراي همسر & \multirow{2}{*}{ وضعيت تأهل } \\
\hline & $r e / I V(1+\varepsilon)$ & $\Lambda r / Q \Lambda(f e)$ & PNEA(IA) & $r \cdot / r q(1 V \cdot)$ & جداشده يا همسرمرده & \\
\hline \multirow{3}{*}{$.1 .8 \mathrm{~V}$} & $r T / r q(I V \cdot)$ & $\Delta T / \Delta F(\& T)$ & $g F / N \&(M Y)$ & $r q / 4 r(194)$ & ابتدايى نهضت & \multirow{3}{*}{ وضعيت تحصيلات } \\
\hline & $r e / N(1 \cdot \theta)$ & $T / / 9(T \Delta)$ & TVEY(A) & TV/ef(Ire) & ديير ستان & \\
\hline & TI/Pr(ITE) & $r e / T r(r))$ & $|r / \Delta\rangle(\Delta)$ & MT/AY(IET) & دييلم دانشكاه & \\
\hline \multirow{2}{*}{.10 .4} & $A Q / F A(M C \cdot)$ & $V \varepsilon /+V(A 9)$ & $V / / F(Y \Delta)$ & $A T / A F(F \Delta F)$ & دارد & \multirow{2}{*}{ مالكيث مسكن } \\
\hline & $1 F / N F(A F)$ & $M T / Q T(Y A)$ & rNAY(1.) & $\mid V / \backslash Q\left(q T^{\circ}\right)$ & ن تلدارد & \\
\hline \multirow{2}{*}{$+1+\infty 1$} & $q 4 / x f\left(m r^{f}\right)$ & $M V \cdot(1 \cdot Y)$ & $N \otimes / M\left(M^{*}\right)$ & $q r / T r(\Delta+\varepsilon)$ & دارد & \multirow{2}{*}{ وضعيت بيمه درمان } \\
\hline & $\Delta / \Delta F(M Y)$ & $11 / r \cdot(1 \%)$ & $1 F / r q(\Delta)$ & $V / \Delta V(F+)$ & 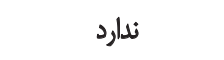 & \\
\hline \multirow{3}{*}{$\cdots \cdots$} & Me/TA(IIV) & 19/Pq(Tr) & $1 f / r q(\Delta)$ & YQ/AF(IEA) & دارد & \multirow{3}{*}{ رضايت اقتصادى } \\
\hline & MT/TA(IRT) & $\Delta \Delta /+\Lambda(8 \Delta)$ & $\Delta V / I F(Y+)$ & $r q / F r(r \mid A)$ & 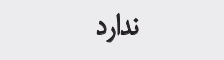 & \\
\hline & $r T / \Delta \cdot(1 Y \cdot)$ & $r A / F r(T \cdot \cdot)$ & YNAY(1.) & $r \cdot / M^{e}(I V \cdot)$ & تاجلودى & \\
\hline \multirow{3}{*}{$.1+.+1$} & $\Delta H / T Y(Y I Y)$ & $r q / q)(r \otimes)$ & $r \Delta / T q(I T)$ & PV/AR(YAQ) & دارد & \multirow{3}{*}{ رضايت ازز زندكى } \\
\hline & $\checkmark / \Delta \Delta(Y \wedge)$ & $r V / T \otimes(T Y)$ & $r e / e v(q)$ & $14 / 49(19)$ & 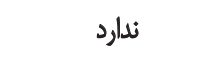 & \\
\hline & $r V / A(I F A)$ & $\operatorname{rT} / M+\left(\Delta_{+}\right)$ & rNIV(IN) & TNAT(YII) & تاحلودى & \\
\hline
\end{tabular}

L

اضطراب بيشترى را در مقايسه با افرادى كه تنها زندكى كردهاند،

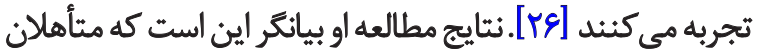

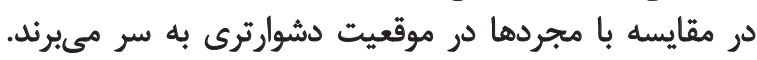

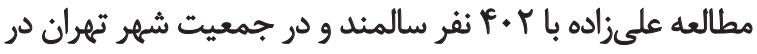

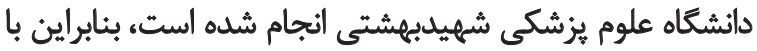

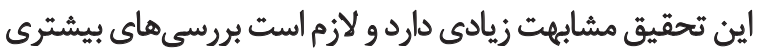

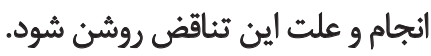

مطالعه حاضر نشان داد افسردگى با مالكيت مسكن رابطه
مشكلات زندكى خود بيدا كنند.نتايج اين بررسى حاكى از وجود

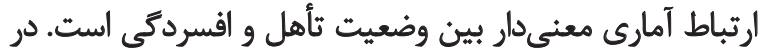

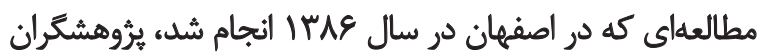

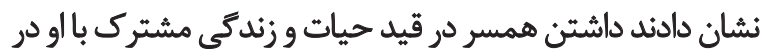

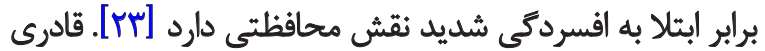

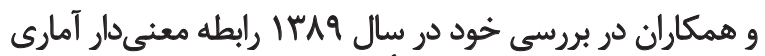

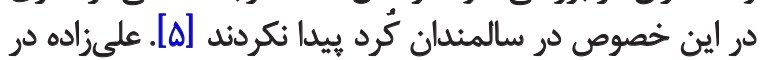

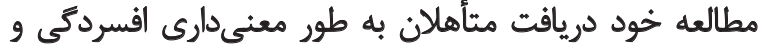

جدول هـ ارتباط حمايت اجتماعى ادراك شده با افسردكى در سالمندان ساكث درمناطق شهرى تهران درسال بهوبا

\begin{tabular}{|c|c|c|c|c|c|}
\hline معنى دارى & دورد (تعداد) & درصد (تعد/د) & درصد (تعداد) & مروصد (تعداد) & \\
\hline \multirow{3}{*}{$+1+\ldots+1$} & 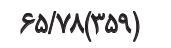 & $v^{c} / \varepsilon q(r u)$ & $\Gamma r / A r(\Delta T)$ & $r+(q)$ & هيج وخفيف \\
\hline & $M / / M\left(W^{Q}\right)$ & $r \Delta / r I(1+1)$ & $\Delta \Delta / I Y(G+)$ & $V+(r I)$ & متوسط و شديد \\
\hline & $\left.1 .(\Delta)^{e} \Delta\right)$ & 1..(พ99) & $1 . .(118)$ & $1 \cdot \cdots(r \cdot)$ & جمع \\
\hline
\end{tabular}


را از يكديكر دريافت كنند. ميتوان اين موفقيت را به بيروزى إزي

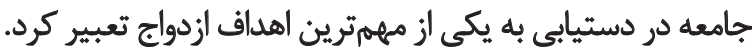

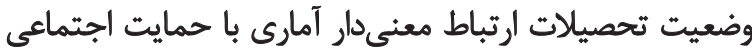

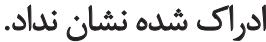
اين بررسى نشان داد ميانكين حمايت اجتماعى ادراكشده

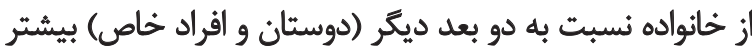

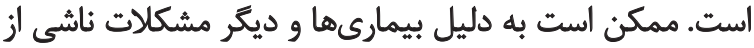

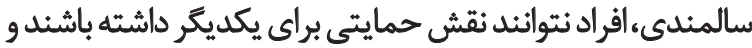

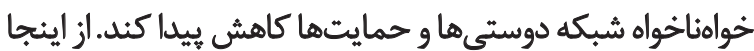

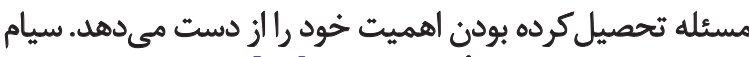
و همكاران اين يافته را تأييد نكردهاند [بrان] داشتن مسكن ملكى و بيمه درمان نيز از عوامل مرتبط با حمايت

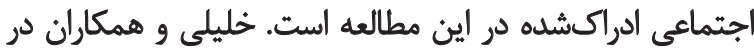

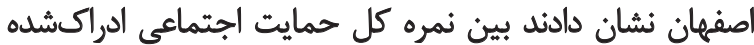

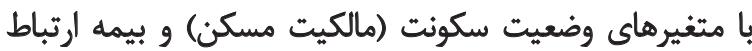

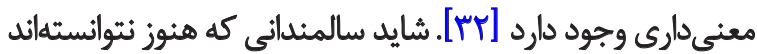

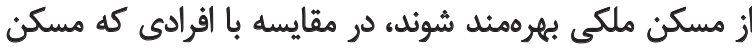

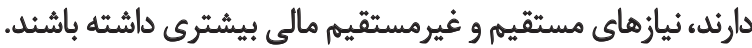

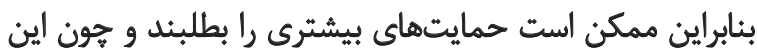

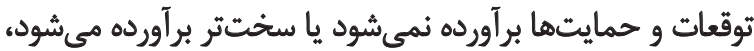

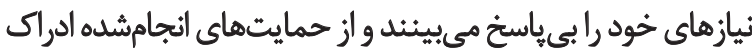

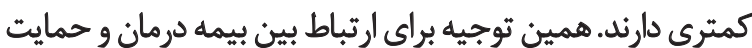

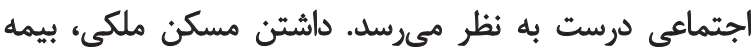
درمان و همه شاخصهايي كه به وضعيت اقتصادى مرتبط درئ است،

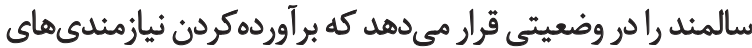

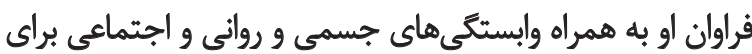

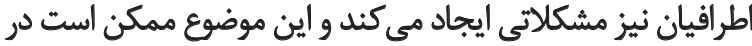

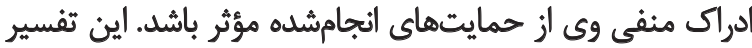

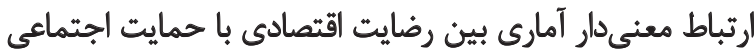

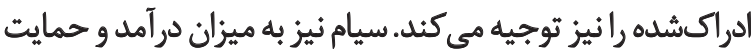
اجتماعى ادراكشده اشاره كرده است [برئ.

بررسى حاضر نشان داد بين رضايت از زندگى و حمايت

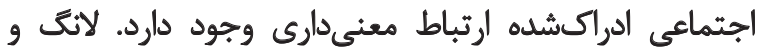

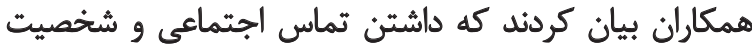

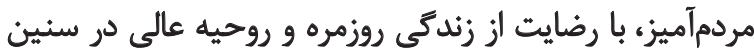

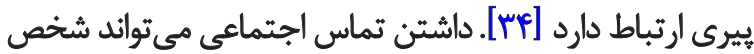

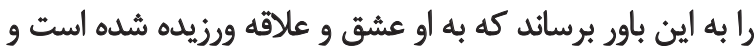

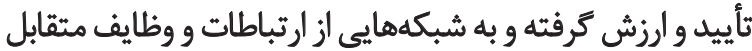

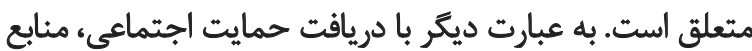

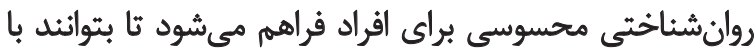

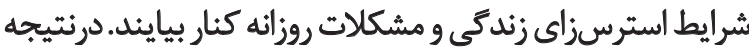

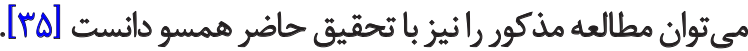
به طور عقلاتى و منطقى مي توان يذيرفت افرادى كه در شبكه
معنى دارى دارد. عليز اده و همكاران نيز در مطالعه خود دريافتند

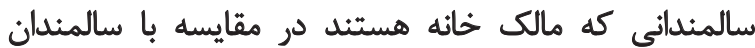

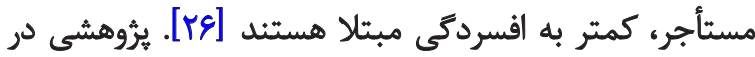

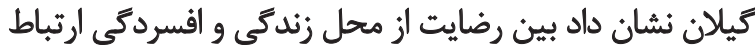

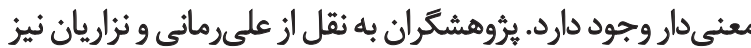

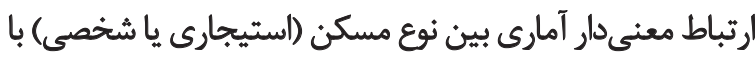

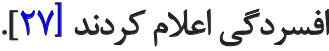

بررسى حاضر ارتباط آمارى معنى دارى را بين افسردگى قريى

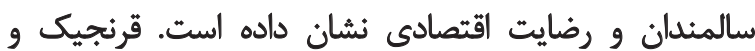

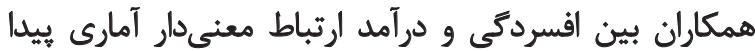

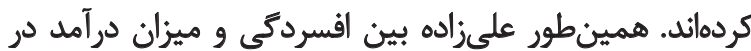

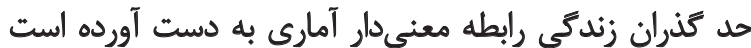

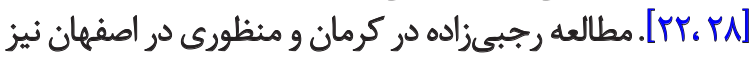

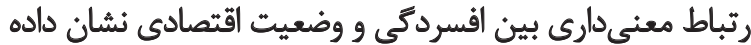

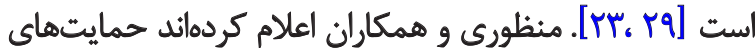

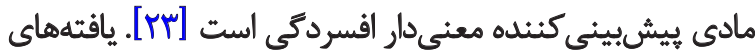

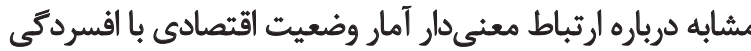

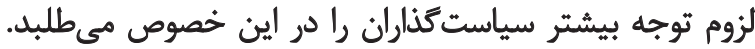

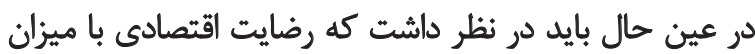

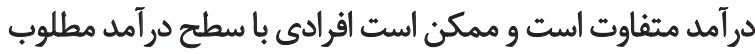

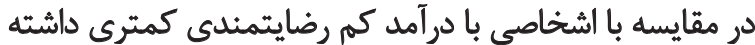

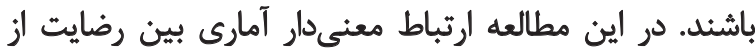

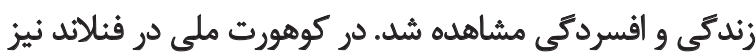

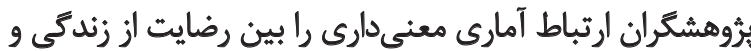

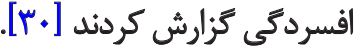

در بررسى ارتباط بين متغيرهاى مطالعهشده و حمايت

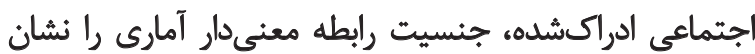

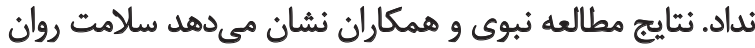

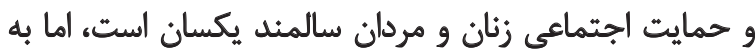

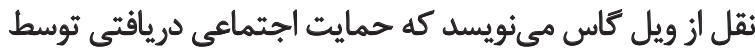

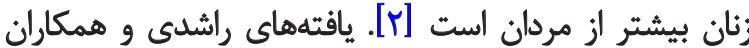

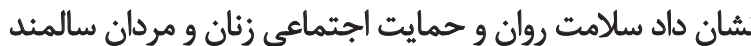

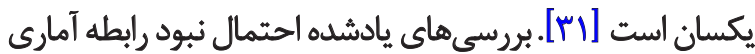

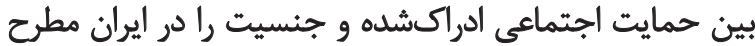

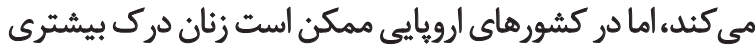

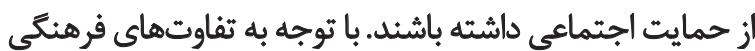

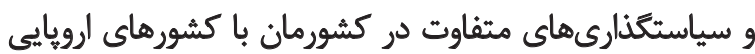

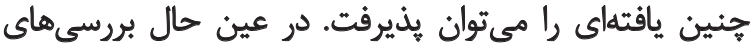
بيشتر لازم است تا علل اين تفاوت روشن شودان

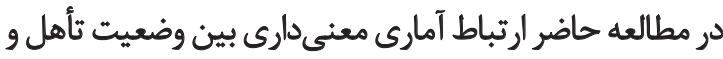

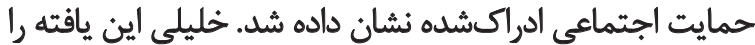

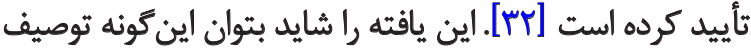

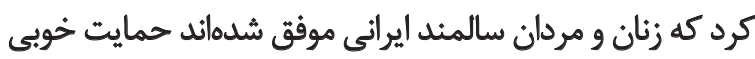


مسئولان با برنامهريزى مناسبتر مىتوانند كارهاى زير را انجام

SOlos

- با ايجاد موقعيتهاى بهتر اقتصادى براى سالمندان، شرايط

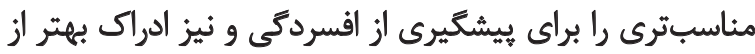

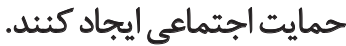

- ازدواجها را تدوام بخشند. ازدواج براى سالمندان از عوامل

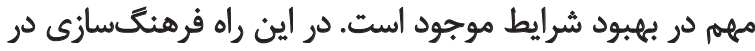

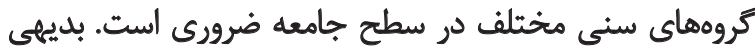

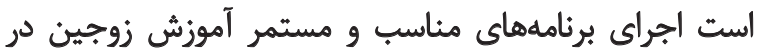

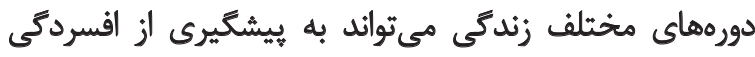
وتداوم زندگى زناشويى موفق كمك كند.

- ايجاد فضاى حمايثكر براى تمام سنين و بلويثه حمايت

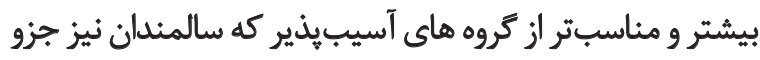
اين تروه هستند.

- براى شناسايى راههاي افزايش سطح رضايتمندى در افراد

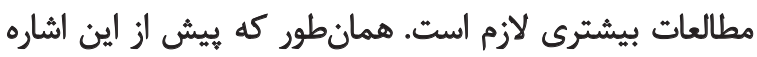

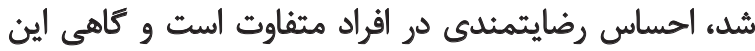

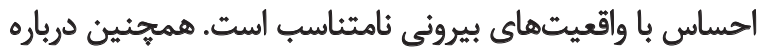

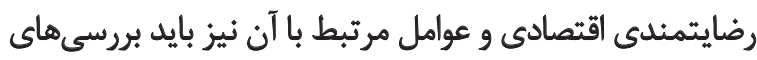

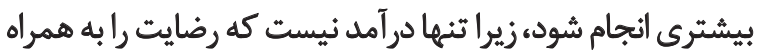

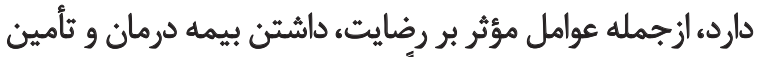

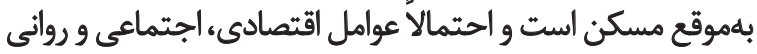
بيشترى در اين ميان نقش دارد كه بايد شناسايى شود. - فعال كردن نظام حمايتى (خانواده، دوستان و همسايكان) و و و

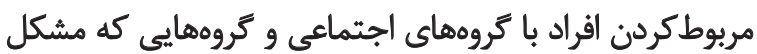

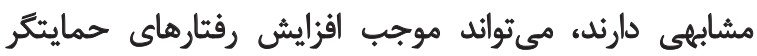

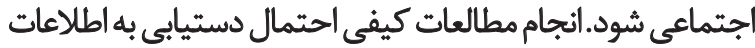
مفيدتر و دقيقتر را فراهم خواهد كرد.

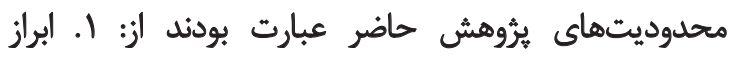

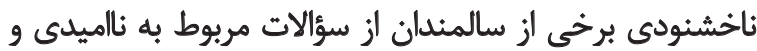

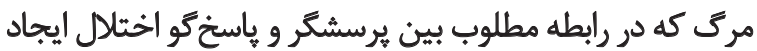

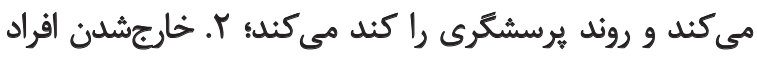

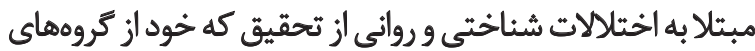
در معرض خطر زياد افسردىى هستند.

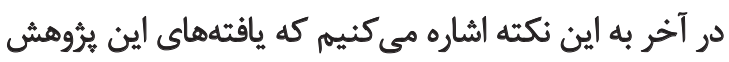

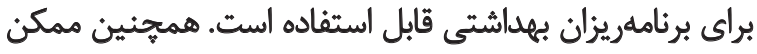
است براى آموزش دانشجويان و اهداف بثروهشى مفيد باشد.

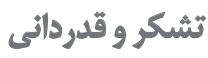

اين مقاله از إياينامه دوره عالى بهداشت عمومى بهاهي نويسنده سوم در دانشكده بهداشت دانشَّاه علوم يزشكى شهيد
ارتباطات خود احساس حمايت كافى دارند، زندكى را راحتتر و با ميا

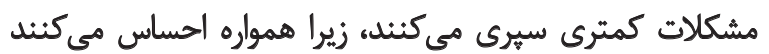

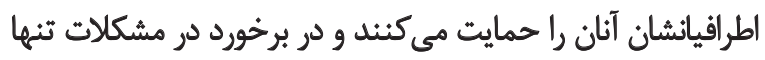
نيستندو بنابراين رضايتمندى بيشترى رادر زئدكى احساس مى كئندان

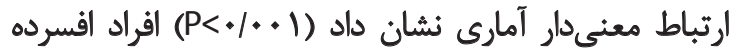

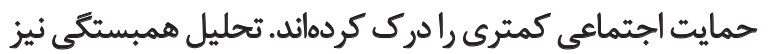

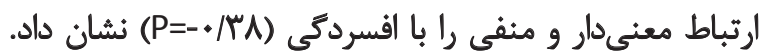

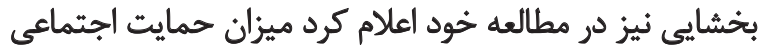

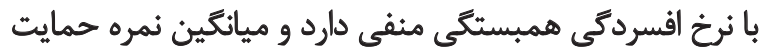

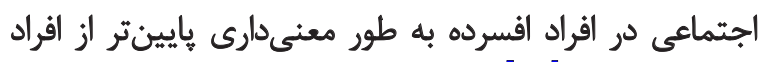

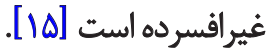

اين ارثباط شايد مؤيد اين نكته باشد كه از آنجا كه افراد افسرده

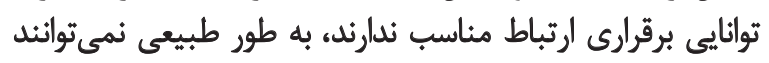

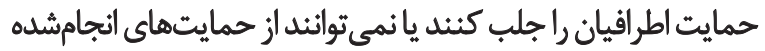

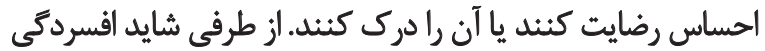

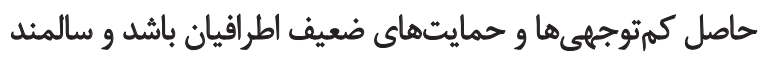

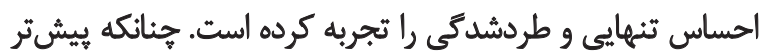

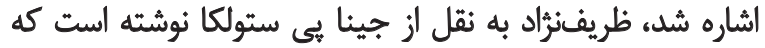

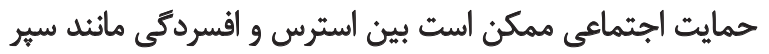

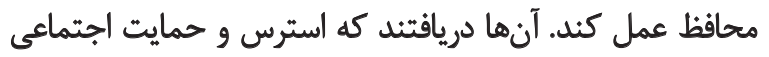

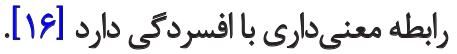

\section{تتيجهئيرى نهايى}

بررسى حاضر نشان داد عوامل مرتبط با حمايت اجتماعى إنى

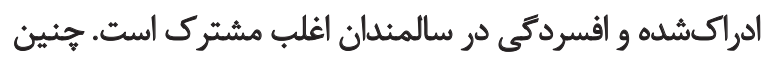

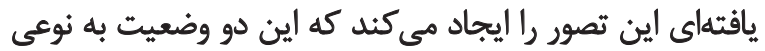

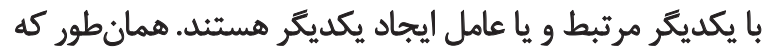

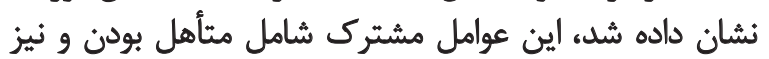

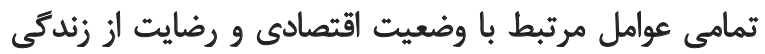

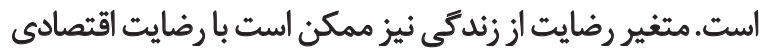

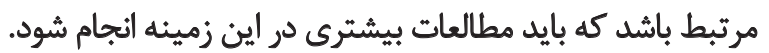

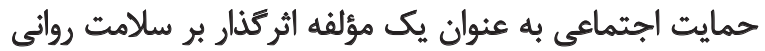

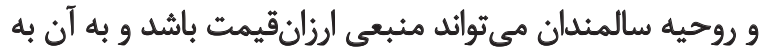

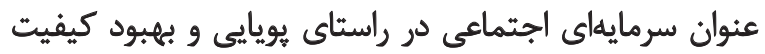

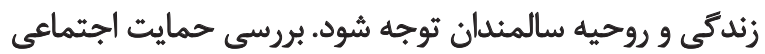

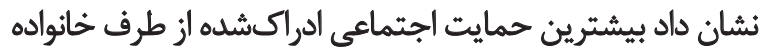

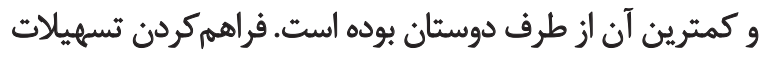

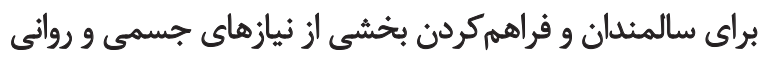

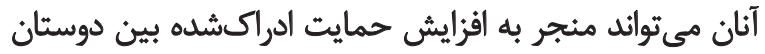

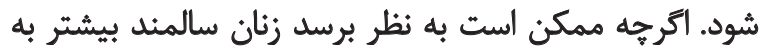

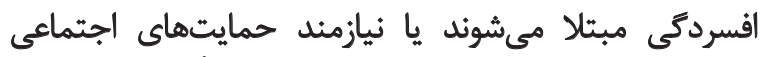

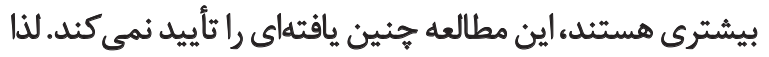
نبايد درباره مردان در اين زمينه غفلت شود. 
بهشتى ترفته شده است. از تمامى افرادى كه در اين برؤوهش

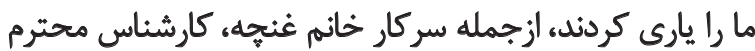

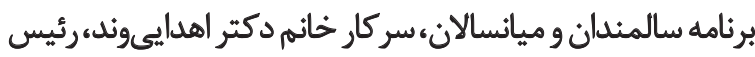

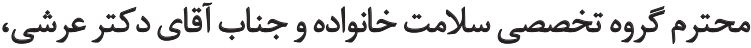

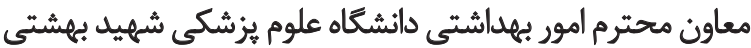

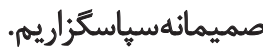




\section{References}

[1] Sadeghi Z. [Opening speech (Persian)]. Paper presented at: The $1^{\text {st }}$ International Conference of Aged People in Iran; 1999 October 19-21; Tehran, Iran.

[2] Nabavi SH, Alipour F, Hejazi A, Rabbani E, Rashedi V. [Relationship between social support and mental health in older adults (Persian)]. Medical Journal of Mashhad University of Medical Sciences. 2014; 57(7):841-6.

[3] Zanjani H. [Opening speech (Persian)]. Paper presented at: The $1^{\text {st }}$ International Conference of Aged People in Iran; 1999 October 19-21; Tehran, Iran.

[4] Bahrami F, Ramezani-Farani A. [Religious orientation (internal and external) effects on aged mental health (Persian)]. Journal of Rehabilitation. 2005; 6(1):42-7.

[5] Ghaderi S, Sahaf R, Mohammadi Shahbolaghi F, Ansari G, Gharanjic A, Ashrafi K, et al. [Prevalence of depression in elderly Kurdish community residing in Boukan, Iran (Persian)]. Iranian Journal of Ageing. 2012; 7(24):57-66.

[6] Centers for Disease Control and Prevention. Healthy aging [Internet]. 2015 [Updated: 2015 March 5]. Available from: http:// www.cdc.gov/aging/mentalhealth/depression.htm

[7] Alipour F, Sajadi H, Forouzan A, Nabavi H, Khedmati E. [The role of social support in the anxiety and depression of elderly (Persian)]. Iranian Journal of Ageing. 2009; 4(11):53-61.

[8] Navvabinejad S. Psycology of aging and mental health in elderly adults. Paper presented at: The $1^{\text {st }}$ International Conference of Aged People in Iran; 1999 October 19-21; Tehran-Iran.

[9] Cheraghi MA, Davari Dolatabadi E, Salavati M, Moghimbeigi A. [Association between perceived social support and quality of life in patients with heart failure (Persian)]. Iran Journal of Nursing. 2012; 25(75):21-31

[10] Hesam M, Asayesh H, ghorbani M, Shariati A, Nasiri H. [The relationship of perceived social support, mental health and life satisfaction in martyrs and veterans students of state university in Gorgan (Persian)]. Journal of Research Development in Nursing \& Midwifery. 2011; 8(1):34-41.

[11] Afrooz Q, Taghizadeh H. [Comparison of perceived social support and mental health of mothers of children with and without hearing-impairment (Persian)]. Journal of Exceptional Education. 2014; 14(124):7-17.

[12] Helgeston VS, Cohen S. Social support and adjustment to cancer: Reconciling descriptive, correlational, and intervention research. Health Psychology. 1996; 15(2):135-48. doi: 10.1037// 02786133.15.2.135

[13] Ghodsi A. [Association between social support and depression in elderly people in Amir Kola city (Persian)] [MSc. thesis]. Tehran: Tarbiat Modares niversity; 2004.

[14] Rahgozar M. [Feelings of depression, stress, and anxiety in the elderly (Persian)]. Hakim Health Systems research journal. 1999; 2(2):103-13.

[15] Bakhshani NM, Birashk B, Atefvahid MK, Bolhari J. [Correlation of social support and negative life events with depression (Persian)]. Iranian Journal of Psychiatry \& Clinical Psychology. 2003; 9(2):49-55.
[16] Zarifnejad G, Saberi Noghabi E, Delshad Noghabi A, Koshyar H. [The relationship between social support and morale of elderly people in Mashhad in 2012 (Persian)]. Journal of Rafsanjan University of Medical Sciences. 2014; 13(1):3-12.

[17] Brummett BH, Mark DB, Siegler IC, Williams RB, Babyak MA, Clapp-Channing NE, et al. Perceived social support as a predictor of mortality in coronary patients: Effects of smoking, sedentary behavior, and depressive symptoms. Psychosomatic Medicine 2005; 67(1):40-5. doi: 10.1097/01.psy.0000149257.74854.b7

[18] Wang JJ. Prevalence and correlates of depressive symptoms in the elderly of rural communities in southern Taiwan. Journal of Nursing Research. 2001; 9(3):1-12. doi: 10.1097/01. jnr.0000347558.69317.66

[19] Papadopoulos FC, Petridou E, Argyropoulou S, Kontaxakis V, Dessypris N, Anastasiou A, et al. Prevalence and correlates of depression in late life: A population based study from a rural Greek town. International Journal of Geriatric Psychiatry. 2005; 20(4):350-7. doi: 10.1002/gps.1288

[20] Scogin F, Beutler L, Corbishley A, Hamblin, D. Reliability and validity of the short form beck depression inventory with older adults. Journal of Clinical Psychology. 1988, 44(6):853-7. doi: 10.1002/1097-4679(198811)44:6<853::aidjclp2270440604>3.0.co;2-7

[21] Jowkar B, Salimi A. [Psychometric properties of the short form of the social and emotional loneliness scale for adults (SELSA-S) (Persian)]. Journal of Behavioral Sciences. 2011; 5(4):311-317.

[22] Alizadeh M, Hoseini M, Shojae zadeh D, Rahimi A, Arshinii M, Rohani H. [Assessing anxiety, depression and psychological wellbeing status of urban elderly under represent of Tehran metropolitan city (Persian)]. Iranian Journal of Ageing. 2012; 7(26):66-73.

[23] Manzouri L, Babak A, Merasi M. [The depression status of the elderly and it's related factors in Isfahan in 2007 (Persian)]. Iranian Journal of Ageing. 2009; 4(14):27-33

[24] Saberian M, Hajiaghajani S, Ghorbani R, Behnam B, Maddah S. [The mental health status of employees in Semnan University of Medical Sciences (Persian)]. Koomesh. 2006; 8(2):85-91.

[25] Luppa M, Sikorski C, Luck T, Weyerer S, Villringer A, König $\mathrm{HH}$, et al. Prevalence and risk factors of depressive symptoms in latest life-results of the Leipzig Longitudinal Study of the Aged (LEILA 75+). International Journal of Geriatric Psychiatry. 2011; 27(3):286-95. doi: 10.1002/gps.2718

[26] Alizadeh M, Khoshbin S, Khavarpour F. Assessing quality of life, well being and depression among Iranian ederly in Australia. Iranian Journal of Ageing. 2007; 4(4):15-26

[27] khodadady N, Sheikholeslami F, RezaMasouleh S, Yazdani M [Rate of depression in late-life in superannuated government employed center of Guilan University of medical sciences (Persian)] Holistic Nursing and Midwifery Journal. 2007; 17(1):16-22.

[28] Gharanjik A, Mohammadi Shahbolaghi F, Sahaf R, Ansari G, Najafi F, Ghaderi S, et al. [Prevalence of depression in Turkmen older adults-1389 (Persian)]. Iranian Journal of Ageing. 2011; 6(21):34-9.

[29] Rajabizadeh G, Ramezani A. [Prevalence of depression in Kermanian geriatrics - 2002 (1381) (Persian)]. Journal of Rafsanjan University of Medical Sciences. 2003; 3(1):58-66.

[30] Koivumaa-Honkanen H, Kaprio J, Honkanen R, Viinamaki H, Koskenvuo M. ife satisfaction and depression in a 15-year follow- 
up of healthy adults. Social Psychiatry and Psychiatric Epidemiology. 2004; 39(12):994-9. doi: 10.1007/ s00127-004-0833-6

[31] Rashedi V, Gharib M, Rezaei M, Yazdani A. [Social support and anxiety in the elderly of Hamedan, Iran (Persian)]. Archives of Rehabilitation. 2013; 14(2):110-5.

[32] Khalili F, Sam S, Shariferad G, Hassanzadeh [The relationship between perceived social support and social health of elderly (Persian)]. Journal of Health System Research. 2012; 7(6):1216-25.

[33] Siam SH. [Elderly issues in Iran and the world (Persian)]. Tehran: Ketab-e Ashena; 2002

[34] Lang FR, Baltes MM. Being with people and being alone in late life: Costs and benefits for everyday functioning. International Journal of Behavioral Development. 1997; 21(4):729-46. doi: $10.1080 / 016502597384640$

[35] Goudarz M, Foroughan M, Makarem A, Rashedi V. [Relationship between social support and subjective wellbeing in older adults (Persian)]. Iranian Journal of Ageing. 2015; 10(3):110-9. 
